THE CORONAL RESPONSES TO THE LARGE-SCALE AND IONG-TERM PHENOMENA OF THE LOWER ILAYERS OF THE SUN

\title{
J. Sýkora
}

Astronomical Institute of the Slovak Academy of Sciences, Skalnaté Pleso, 05960 Tatranská Lomnica, Czechoslovakia

Based on the assumption, generally accepted over the past decade, that all the forms of solar and interplanetary activity are responses to the magnetic fields generated initially in the subphotosphere, some characteristics of the large-scale and long-term behaviour of the solar corona during the last three solar cycles are presented.

Most part of our report is based on the statistical and graphical processing of the abundant observational material pertinent to $530.3 \mathrm{~nm}$ Fe XIV coronal line. Apart from these, some white-light corona and EUV observations were used to demonstrate the long-term phenomena. Namely, the longitudinal and latitudinal distributions of the corona brightness are shown and, in this connection, active longitudes and coronal rotation are discussed. Some solar cycle properties and N-S asymmetry are compared with similar features found for sunspots and solar flares. Large-scale characteristics and longterm evolution of coronal holes are briefly summarized.

\section{INTRODUCTION}

Although our symposium is focused primarily on short-term phenomena on the Sun and their effects in the interplanetary medium, the Scientific Organizing Committee felt that it was also necessary to describe the recent understanding of the "background" solar and interplanetary medium characteristics. We will attempt to do this for the solar corona.

It is certainly correct to follow idea, generally accepted over the past decade, that all the forms of solar and interplanetary activity are consequences of magnetic fields, generated initially in the subphotosphere, and that it would be difficult and misleading to study either short-lived or long-term coronal phenomena apart from their connection with photospheric magnetic fields. Several fundamental introductory papers giving reasons for this point of view were presented at the last solar symposia (e.g., Newkirk, 1971; 
Wilcox, 1971; Altschuler, 1974; Pneuman, 1974; Bumba, 1976). Therefore, taking into account the number of papers referred to in the mentioned summarizing papers, speaking of coronal responses to longterm phenomena on the Sun means, above all, to discuss responses to long-term and large-scale phenomena of the photospheric magnetic fields. Of course, in analysing the distribution of coronal brightness through the solar cycle, N-S asymmetry and coronal rotation we will also frequently make comparisons with the distribution of sunspots and solar flares, the association of which with magnetic fields is indisputable.

It is clear that responses both to short- and long-term phenomena do not stop in the solar corona. They are transported through and certainly transformed by this layer and proceed into the interplanetary medium to the upper and lower layers of the Earth's atmosphere and beyond. Viewed in this way, the study of the large-scale behaviour of the solar corona should prove a good tool to understand disturbances in terrestrial magnetism, the ionosphere and possibly the influences on life in the troposphere. But this topic is certainly closer to the speaker who will follow me.

During the last few years the observational material enabling us to study the individual physical processes in the corona, on the one hand, and statistical large-scale manifestations of coronal activity, on the other hand, has markedly increased. This is not only as a result of space research, but also thanks to the systematic, routine ground-based observations.

Our report is primarily based on the statistical and graphical analysis of the abundant observational material obtained by the network of corona stations, headed by Pic du Midi, which observe the monochromatic corona, particularly in the green $(530.3 \mathrm{~nm})$ and red $(637.4 \mathrm{~nm})$ lines. A detailed description of the data used and of the process of their homogenization is given in (Sýkora, 1971a, 1971b). These data refer to the height of about $43500 \mathrm{~km}$ above the photosphere and their resolution on the Sun's surface is quite small - one day in heliographic longitude and $5^{\circ}$ in heliographic latitude - but for studying the large-scale phenomena this even seems to be an advantage. The green corona data are used in our study because they are fairly complete over sufficiently long period of time and as we shall see further on, they allow us to draw some conclusions on the corona as a whole (i.e. on the corona observed in the X-ray, optical and radio intervals).

We should also mention what is meant by large-scale and long-term phenomena in this paper. Large-scale means that the dimensions of the investigated features are greater than the scale of one active region and long-term refers to features with a duration of one solar rotation to one solar cycle, nevertheless, the last three solar cycles will be compared. 


\section{IONGITUDINAL DISTRIBUTION OF THE GREEN CORONAL EMISSION}

Most of the solar magnetic field features we are interested in association with long-term coronal responses were obtained from an all-round study of the synoptic charts constructed from daily lowresolution magnetograms of the Mount Wilson Obsevatory. The charts were partially published as the "Atlas of Solar Magnetic Fields 1959-1966" by Howard et al. (1967). The analysis of the separate polarities allowed to Bumba and Howard (1969) to postulate certain new patterns in the large-scale field distribution - sections, rows and streams. These are features, the width of which is some tens of degrees in latitude, and they clearly show that the activity distribution on the Sun's disk is not regular, but, on the contrary, during long periods - as long as several years - the activity in certain longitudinal intervals is higher in comparison with other heliographic longitudes. A very well pronounced property of Bumba's and Howard's formations is their recurrence period which is close to 27 days, in higher latitudes 28-29 days (see also Bumba, 1976).

Similar regularities, in most studies called "active longitudes", have been found by many authors for different types of solar phenomena. But the understanding and definition of active longitudes differed from author to author and, so far, only a few attempts have been made to identify the "active longitudes" of the various phenomena.

Also we can comment only qualitative similarity (response) of the green corona longitudinal distribution to that of the magnetic field. Examples of four times repeated 27-day sequences of daily data on green corona brightness, prepared in a way similar to that described in (Sýkora, 1971b), are shown in Figure 1. Originally, the data from the period 1947-1976 were processed separately for every $5^{\circ}$ in the interval of $\pm 60^{\circ}$ of solar latitude. In this figure only data for every $10^{\circ}$ of the northern hemisphere are presented. Variations of activity with the solar cycle were subtracted. It can be clearly seen that the activity in the last three solar cycles is not distributed randomly, but on the contrary, this distribution holds almost all the characters of the active longitudes as they were postulated in the past and reminds many properties (width, duration, inclination) found by Bumba and Howard for the background magnetic fields and perhaps even more so of the sector structure of the interplanetary magnetic field as it is seen for example in Figure 10 of Bumba's paper in IAU Symp. No.71 (Bumba, 1976). The different inclinations seen in our Figure 1 are mainly connected with coronal rotation and differential rotation and we shall discuss them in section 4 .

From the point of view of solar-terrestrial relations, in recent years the coronal holes and, generally speaking, the low-brightness coronal regions seem to be very important. It is clear, that Figure 1 shows the distribution of these features just as well, if the bright area of the figure is analysed. 


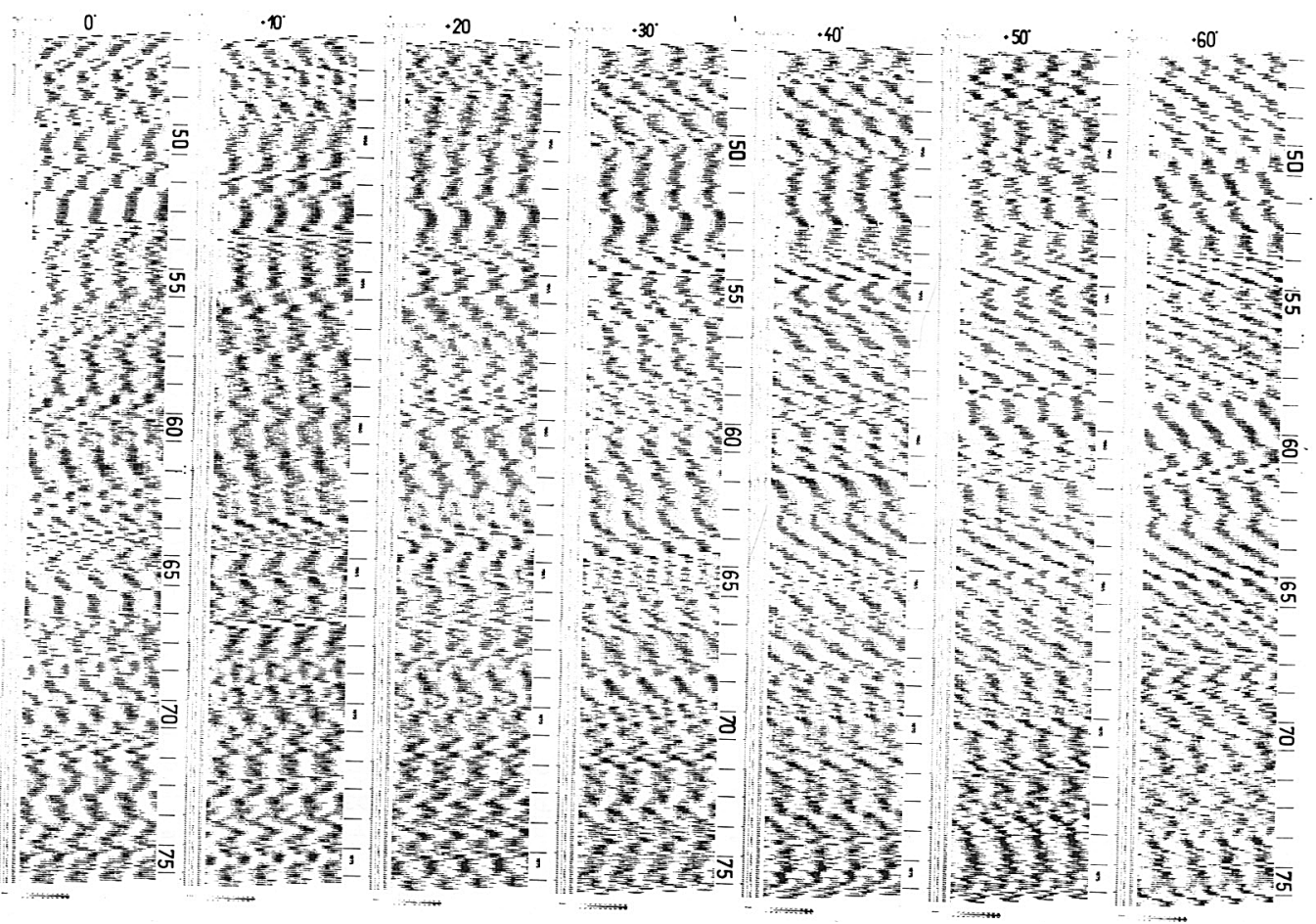

Figure 1. Four times repeated green corona brightness observed in discrete latitudes is visualized in succession of Bartels rotations over the last two and a half solar cycles.

The green corona synoptic tables we prepared for the period 1947-1970 have been extensively used by Antonucci and her co-workers for an all-round study of the large-scale distribution of the coronal emission. She found (Antonucci, 1974) that enhancements of the green corona show an organized pattern within solar magnetic sectors. Antonucci and Svalgaard (1976) found correlation peaks amplitude in excess of 0.1 between corresponding northern and southern latitude zones. They conclude: "The very fact that any correlation exists between corresponding latitude zones in opposite hemispheres at a lag significantly different from zero for these 24 yr time series, strongly suggest that the green line corona includes a component which is organized on a very large scale." The high correlation between northern and southern high-latitude emissions at a 15-day time lag is explained as a feature of the two-sector solar magnetic structure, while four sectors are associated with 6- and 24-day peaks.

As an interesting response to the long-term distribution of the photospheric magnetic fields some years ago we have found (Bumba and Sýkora, 1973; Bumba and Sýkora, 1974) that the maxima of the coronal emission coincide in position with the negative (at least during the declining phase of the 19th cycle and during the increasing phase 
of the recent cycle of solar activity) and the minima with the positive polarity of the photospheric magnetic field. Stenflo's (1972) assumption that the green line intensity should not be related so much to the polarity pattern of the field, but more directly to the field strength is based on the comparison of the very low resolution butterfly diagram of the green line corona for the period 1959-1970 with a similar diagram of magnetic field.

\section{LATITUDINAL DISTRIBUTION OF THE GREEN CORONAL EMISSION}

The latitude-time variation of corona brightness can be illustrated in various ways. Charts of isophotes were used, for example, by Trellis (1957), Waldmeier (1957a), Stenflo (1972), etc. All these diagrams seem to be rather smoothed, revealing only little of the details. In Figure 2 we present similar diagrams for the last three solar cycles. The coronal data, as published in the Quarterly Bulletin on Solar Activity, are expressed in physical units, i.e. in millionths of the energy radiated from the centre of the sun's disk in $0.1 \mathrm{~nm}$ strip of the spectrum near the $530.3 \mathrm{~nm}$ coronal emission
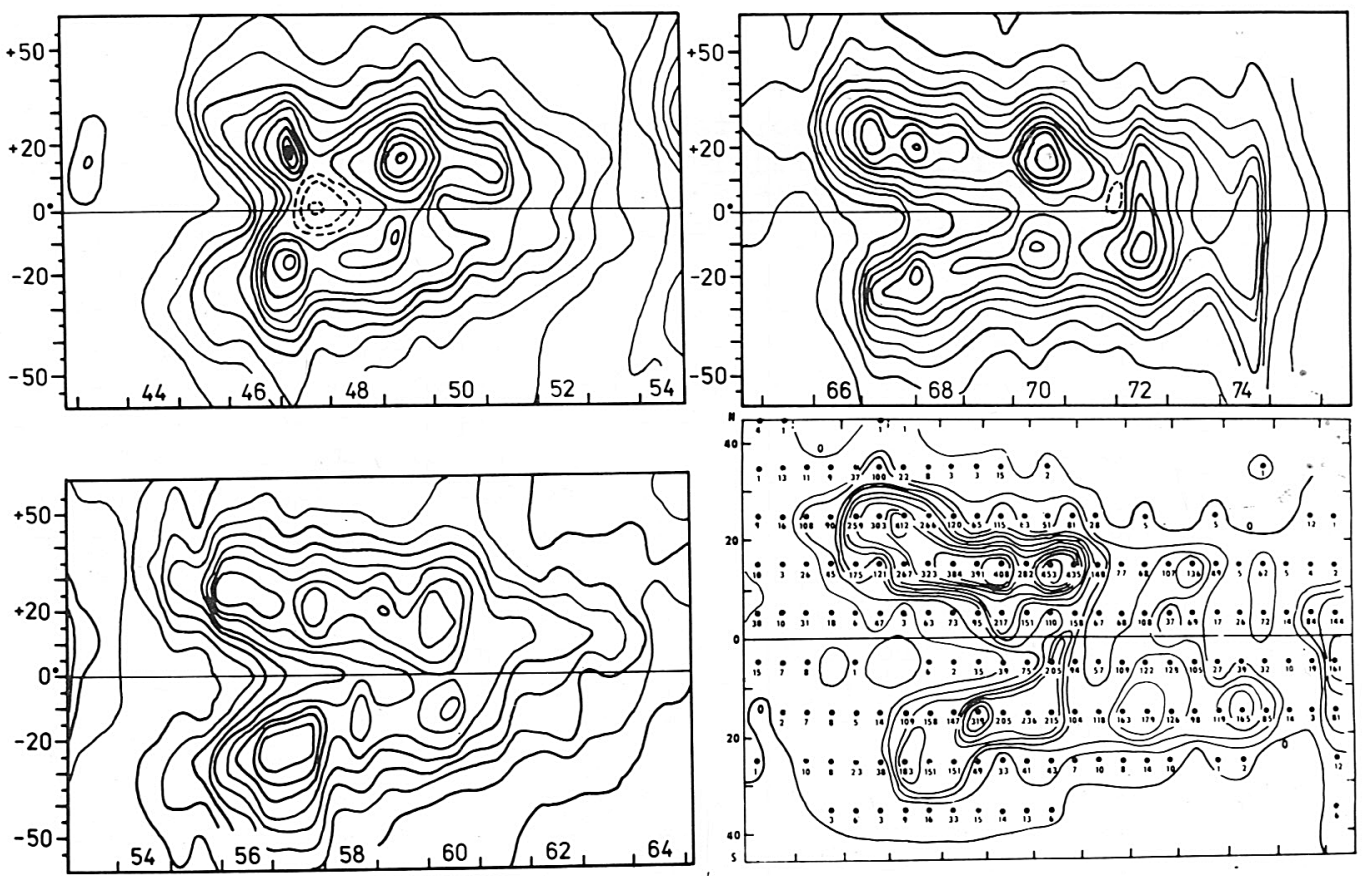

Figure 2. Latitude distribution of the intensity of the green line $(\lambda 530.3 \mathrm{~nm}$ ) corona in the last three solar cycles. The lowest contour level is 15 absolute coronal intensity units and the contours are given with step of 10 units. In the lower right part a similar distribution of the flare occurrence is shown. 
Iine. The homogenized data of various corona stations (Sýkora, 1971b) were averaged over the intervals of six Bartels rotations for each $5^{\circ}$ of solar latitude. We omitted the data for the polar regions. In the lower right-hand part of the figure the diagram of occurrence of flares in the 20 th cycle (Knoška et al., 1978) is shown for comparison with the coronal data.

The following characteristics can be seen in Figure 2: 1) There have place remarkable differences - as large as one or one and a half year - in the beginnings of the cycle in both hemispheres. 2) The fact that activity during cycle consists of several large impulses, on an average two years apart, seems to be realistic. In the previous cycle the impulses in 1967, 1970, 1972 and 1974 were well expressed. The cores of impulses gradually shift in latitude from about $25^{\circ}$ at the beginning to about $10^{\circ}$ at the end of the cycle. There is no evident correlation between their time occurrence in both hemispheres. 3) Because the intensity of the green line increases with coronal temperature, Stenflo (1972) found that the latitude distribution of the green line intensity follows closely that of sunspots, faculae and strength of magnetic fields. On the right-hand side of Figure 2 a comparison can be made with flare occurrence for the 20 th cycle. We should mention that the flares were not weighted for importance. The good agreement between both diagrams is broken at the beginning of 1971, when the strong decrease of the number of flares till the end of the cycle is not associated with a similar decrease in the coronal emission. This fact will be discussed in analysing Figure 6 .

The butterfly diagrams, constructed from points in which the green line intensities, everaged over the single Bartels rotations, reached maximum values, reveal two interesting features (see Figure 3). Firstly, the "butterfly wings" in the 19th cycle extend $10^{\circ}$ further into higher latitudes than in cycles 20 and 18 (see also Figure 9). We have adopted a tentative explanation that in the higher cycles (characterized by the higher sunspot numbers) the activity starts at higher latitudes. Secondly, although the coronal activity is more extended in latitude than the activity of the photospheric and chromospheric phenomena, the highest brightness is only infrequently characteristic of the equator. Not even the summation of the coronal activity from both hemispheres at the end of the cycles necessarily leads to the highest activity at the equator. Only the 20 th cycle perhaps shows such a behaviour.

Another view of the latitudinal distribution of the green line corona brightness during the last two cycles is presented in Figure 4. Here, the distribution of the average radiance at $5^{\circ}$ increments of latitude, is shown for a succession of Bartels six-rotations. During the solar minima of 1954, 1964 and 1976 (curves Nos. 1, 1 and 28) the brightness is practically uniform over all latitudes between $\pm 60^{\circ}$ and reaches $10-15$ absolute coronal units. Responses to the running solar cycles are as follows: At the beginning the activity starts (namely in the 19th cycle) at latitudes of $30-35^{\circ}$, then it 


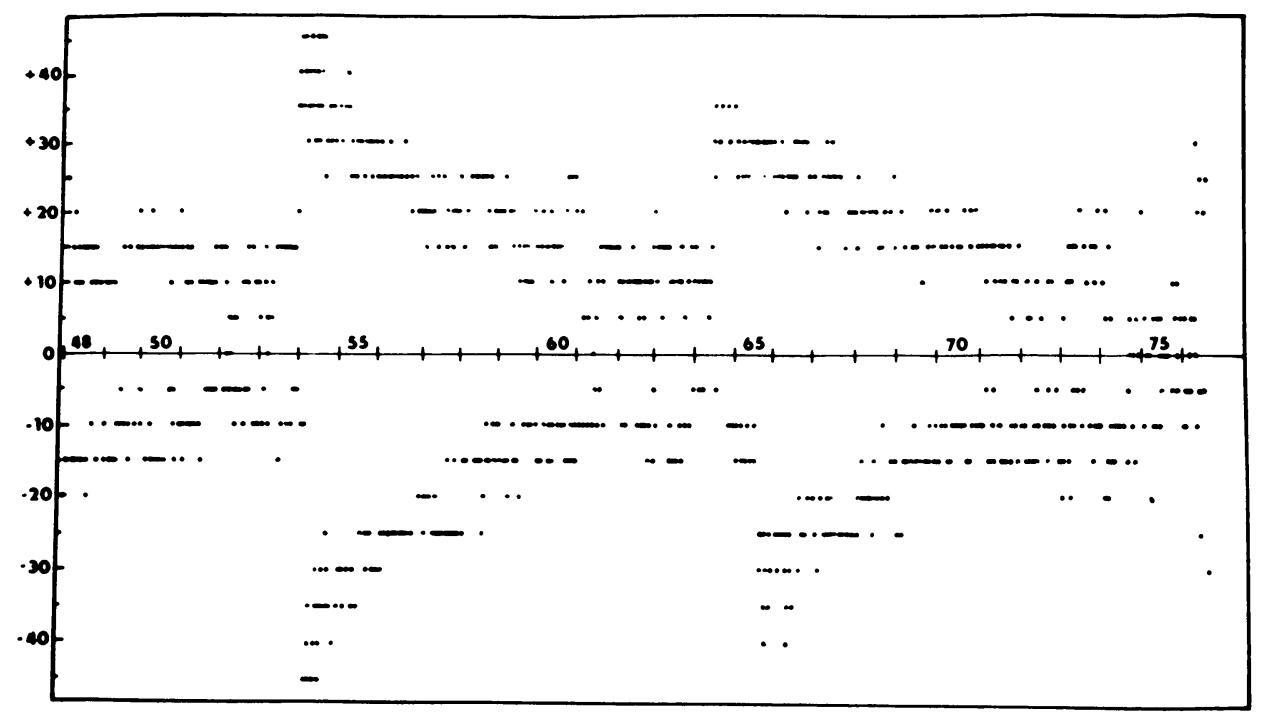

Figure 3. Butterfly diagrams of the green corona intensity for the last two and a half solar cycles.

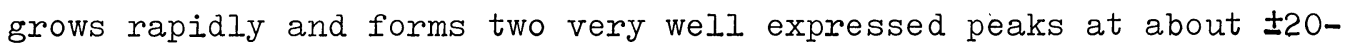
$-25^{\circ}$ in the cycles maxima. The brightness in this period is about ten times higher than that in the solar minima. This fact is in severe contradiction with the maximum-minimum ratio in brightness of the K-corona as published by Hansen et al. (1969a) in their Table II (data from four sources) where this ratio is found to be $2.0-2.5$. The disagreement certainly indicates a much higher sensitivity of the green line intensity to complexity of magnetic fields on the solar disk. Towards the cycle minima the activity regularly migrates to the equator, to latitudes of $\pm 5-10^{\circ}$ and sometimes (as in 20th cycle) the mutual overlapping of the activity from both hemispheres results in the impression that the brightness maximum is located at the equator.

Hansen et al. (1969a) state that the arithmetic averages, as shown in our Figure 4, tend to obscure the fact that the coronal features occur in discrete latitude zones. In Figure 5, adapted from their paper on the K-corona, it is seen clearly that the statistical standard deviation of the intensity from six-months mean, except of brightness maxima well associated with sunspot and plage regions, reveals the high-latitude or polar zone of activity at $60-70^{\circ}$ as they were perhaps firstly pointed out by Trellis (1957). Hansen et al. show that these high-latitude zones steadily migrated to the poles over period 1964-1967 which fact also is known from the movement of prominences and filaments as well as from theory and measurements of solar magnetic fields. We did not attempt to find these polar zones of activity in our green-line data because they are limited by $\pm 60^{\circ}$ in latitude. 
CYCLE No.19

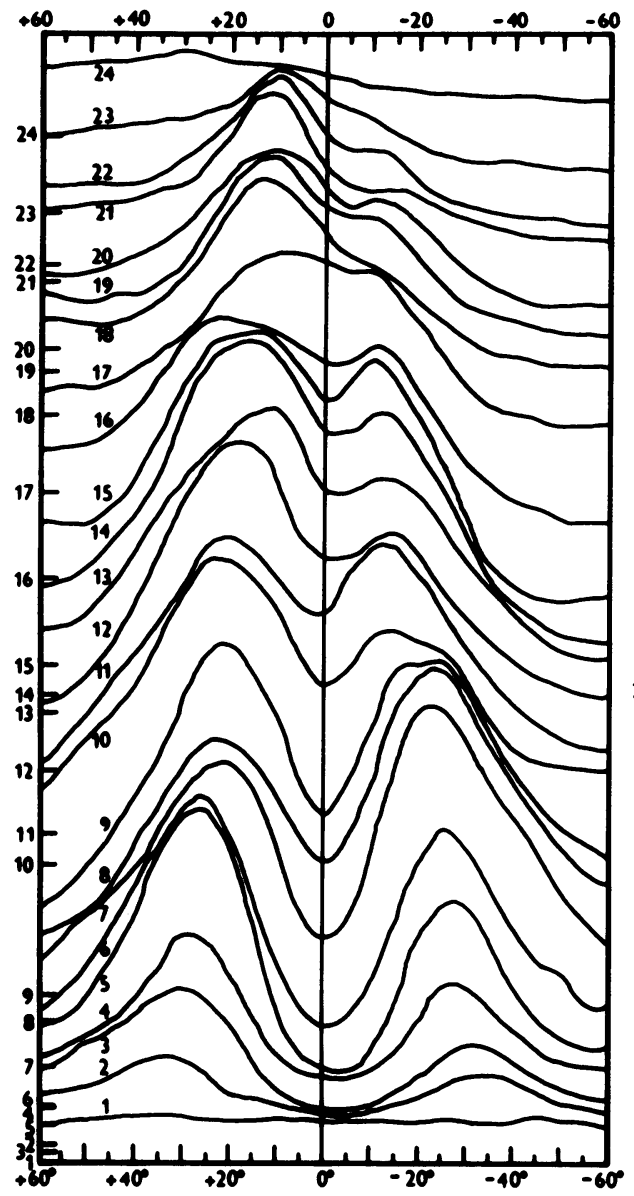

CYCLE NO. 20

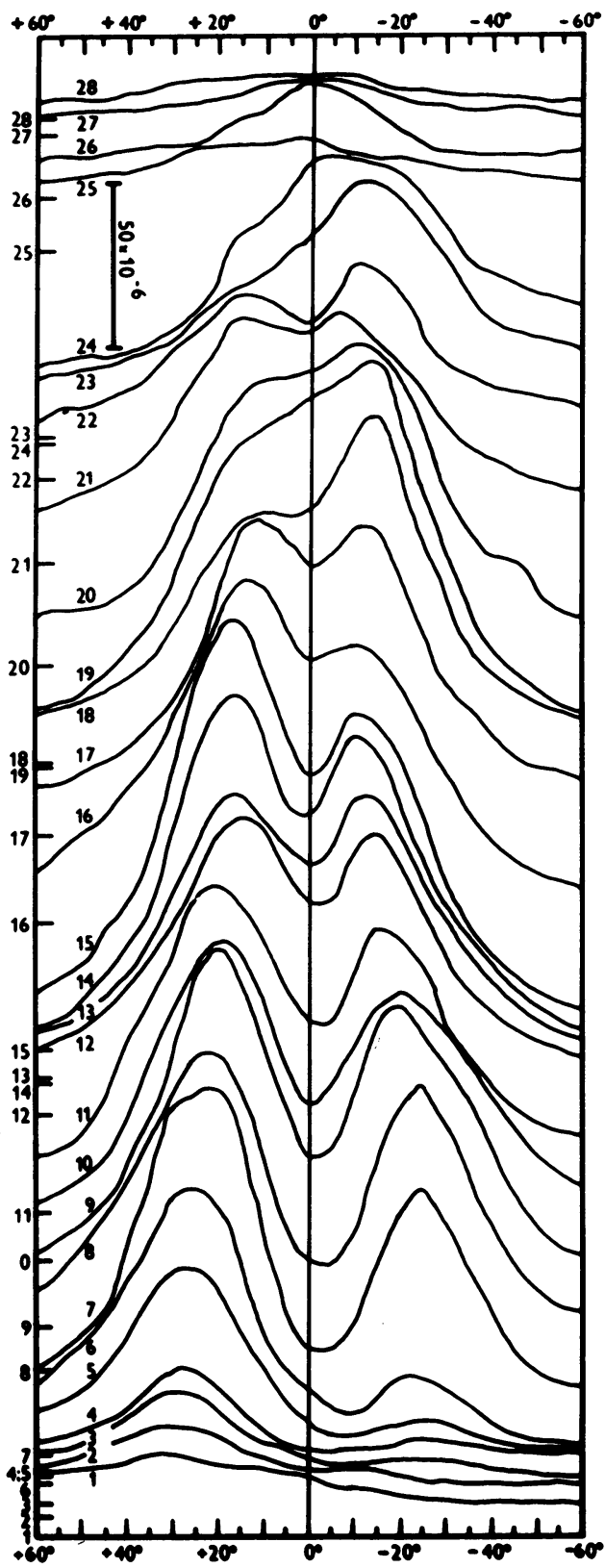

Figure 4. Average brightness distribution of the green corona for Bartels six-rotations (denoted by numbers 1 to 24 and 1 to 28). Number 1 in the cycle No.19 denotes six-rotation 1652 (beginning on Feb. 25, 1954) - 1657 (ending on Aug. 5, 1954), and number 1 in the cycle No.20 denotes six-rotation 1790 (beginning on May 9, 1964) - 1795 (ending on Oct. 17, 1964). Zero points of the curves and the scale in coronal units are given. 


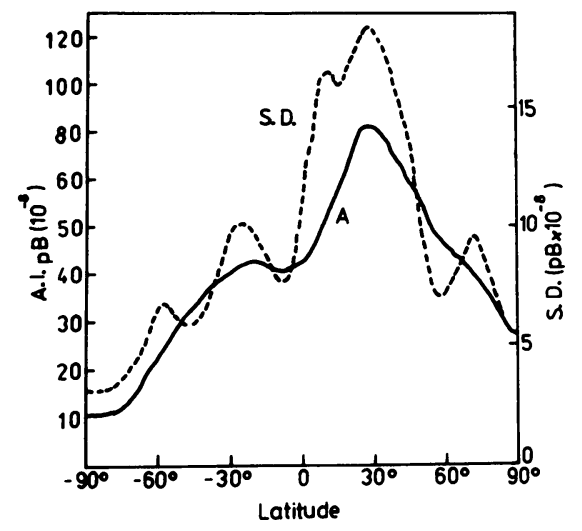

Figure 5. Comparison of the latitude distribution of average K-corona brightness (A) and standard deviation (S.D.) from this mean for the period July - December 1966. - 1.125 Ro (from Hansen et al., 1969).

\section{CORONAL ROTATION}

Much work has been done in the study of the coronal rotation and its latitude-dependence. The results of the past decade seem to be convincing enough with regard to the variety of the analysed data and with respect to quite good agreement among them. Determination of rotation rates is possible because of the existence of the regions (enhanced or quiet, bright or dark) which reappear for several rotations with sufficient stability.

Hansen et al. (1969b) applied an autocorrelation analysis to the limb observations of the white-light corona during 1964-1967. At low latitudes the corona was found to rotate at the same rate as the sunspots, but at higher latitudes it was consistently faster than the underlying photosphere - at the same time at latitudes 60$-65^{\circ}$ it amounts to $12.8^{\circ} /$ day (period of 28.13 days). There were differences as large as $3-4 \%$ in the rate at specific latitudes from year to year and between the two hemispheres. No obvious relation of these changes to increasing solar activity in the studied period has been found.

To our knowledge the paper of Hansen et al. (1969b) is the only one in which the coronal rotation was studied at different height levels using the same observational data. In 1967 a nearly constant rotation was found for heights ranging from 1.125 to $2.0 \mathrm{R}_{0}$. For 1966 there was a more complicated pattern of height dependence, with the rate generally decreasing with height at low latitudes and increasing at high latitudes.

A graphical study of the green-corona synoptic tables, divided into six latitude zones $20^{\circ}$ in width and covering the period 1947- 
1968 showed only little latitude dependence of the rotation rate (Sýkora, 1971b). These rates were derived from the recurrence of the long-lived large-scale active features and the average values of the rotation period are 27.80 days for the $+7.5^{\circ}$ zone and 28.23 days for the $+47.5^{\circ}$ zone. If one was to judge just by the slopes of the strips of activity seen in Figure 1 of the present paper, one should notice the rather complicated pattern of these slopes. If we neglect the changes of the coronal structures, especially those occuring in the longitudinal direction, then the slopes of the strips of activity are indicators of the rotation rate at given time and latitude. Generally, two tendencies of the slopes can be seen. At lower latitudes (from $+10^{\circ}$ to about $+30^{\circ}$ ) almost vertical formations, which are only occasionally interrupted by formations characterized by sufficiently larger slopes, dominate. The large slopes are more pronounced in higher latitude zones $\left(+40^{\circ}\right.$ to $\left.+60^{\circ}\right)$. But at these latitudes the vertical formations are still visible and perhaps they are indicators of "almost rigid" coronal rotation, discussed below.

This very qualitative picture of coronal rotation can be well fitted to Bumba's results on regularities in the large-scale distribution of the solar magnetic fields (Bumba, 1976), especially to the features, the recurrence period of which is 27 and 28-29 days.

The green corona synoptic tables (Sýkora, 1975), mentioned above, were later processed by autocorrelation techniques, and Antonucci and Svalgaard (1974) and Antonucci and Dodero (1977) have published some interesting results. They deduce that the short-lived coronal features (observed in green line $530.3 \mathrm{~nm}$ of Fe XIV), associated with the activity, rotate differentially, while features with a lifetime of the order of about one year or more and characteristic preferably of the years of declining solar activity do not show differential rotation, but instead all rotate in $\approx 27$ days, independent of latitude. These results were also extended to the decreasing phase of the last cycle (Antonucci and Dodero, 1977, 1979).

An effect, similar to that just mentioned, was found earlier in an analysis of the photospheric magnetic fields by Bumba and Howard (1969) and also by Wilcox et al. (1970). The rotation curve of the photospheric magnetic field polarity, computed for period 1959-1970 by Stenflo (1974) is in good agreement with the average corona rotation curve (for comparison see Figure 6 in Antonucci and Dodero, 1977). In addition, Stenflo (1977) has found that the foot points of the large-scale rigidly rotating pattern of the corona are very likely associated with the long-lived component of the photospheric magnetic field. Thus the rotational behaviour of the photospheric magnetic fields and corona are the same, averaging over a solar cycle and, hence, Antonucci and Dodero conclude, that "at present, on analogy of the corona, a variation of the degree of differential rotation of large-scale photospheric magnetic fields through a solar cycle, can not be excluded." 
Another noticable result, presented in the lower part of Figure 4 in the paper of Antonucci and Dodero, is very good coincidence of the rotational characteristics of the EUV (Fe XV, $28.4 \mathrm{~nm}$ ) coronal holes detected by OSO-7 spectroheliograph from May 1972 to October 1973 and analysed by Wagner (1975) with a quiet corona rotation curve. Hence, the rotation characteristics of the corona and coronal holes are very probably the same during low activity periods, and low emission regions probably coincide with coronal holes (see also Letfus et al., this Symposium).

The results on coronal rotation discussed so far have been criticized by Timothy et al. (1975) in the sense that they may heavily be dependent on statistics in which the changes in the coronal structure, particularly those occuring in the longitudinal direction, introduce uncertainties into the analysis of the individual features. It is possible to agree with this opinion, but anyway the result obtained by Timothy et al. is in quite good agreement with the criticized results. They studied the rotation rate of the elongated coronal hole CH1 of the Skylab mission. During five successive rotations an "almost rigid" rotation of this hole has been observed.

Figure 7 in Bohlin (1977) clearly shows that also the rotation of other holes of the Skylab period (except of unusual case of CH5, which was more likely due to intrinsic evolution rather than rotation) confirm the results of Timothy et al. But because features $2^{X}$ and 4 are too low in latitude Bohlin calls for further observations to prove the validity of the "almost rigid" rotation equation $\omega \simeq(13.3 \pm 0.1)-0.4 \sin ^{2} \varphi$.

In summarizing the coronal rotation data one should take into account that the results were obtained from the analysis of quite different data - K-corona, emission corona and coronal holes. The results are sufficiently similar to conclude that at least long-lived large-scale coronal features display a substantially smaller differential rotation than derived from a number of photospheric and chromospheric phenomena. The fact that the results are consistent with the hypothesis on a rigidly rotating "subsurface source", mentioned in Bumba and Howard (1969), and in some other papers on largescale solar magnetic field structure and "active longitudes", is very important.

Of course, the situation is not so simple if we go into details. It is sufficient to look once more at Figure 1 to realize how complicated the rotation rate can be within the cycle and in dependence on latitude. Perhaps it depends on the actual distribution and phase of the development of the large-scale magnetic structures with a lifetime of about 10-20 rotations. Sometimes we see symptoms of rigid rotation, at other times we can not be sure about this. The physical causes of such behaviour are quite obscured. 


\section{SOIAR CYCLE CHARACTERISTICS}

Perhaps we can start this section by presenting the runs of two global characteristics - the sunspot number and the $530.3 \mathrm{~nm}$ coronal line intensity averaged over $\pm 60^{\circ}$ and six Bartels rotations (see Figure 6). The correlation between these two phenomena through the solar cycles does not seem very good. First of all, the relatively equal heights of the coronal cycles are accompanied by the sizable differences in the heights of sunspots cycles. One can obtain the impression that during the cycle the corona reaches some degree of saturation (in the sense of particles), without any further possibility to increase its brightness (19th cycle). The conspicuous decrease of the number of the sunspots after 1970 is accompanied by only a small decrease of the coronal intensity. At the same time two sharp coronal maxima in 1972 and 1974 correspond to the presence of the well-known active complexes in these years which also produced huge proton flares. At the same time, complicated, closed magnetic structures, characteristic of the regions with large flares, serve as a trap for the coronal plasma particles, a long-term enhancement of plasma concentration takes place and this results in long-term increase of coronal brightness in these regions. The number of sunspots does not seem to be important. Similar facts were analysed and quantitatively well expressed by Xanthakis (1969). He found that, for the long-time variation of the coronal intensity, the area of sunspots and faculae and the number of proton flares are far more important characteristics than the relative sunspot number. Frequency of occurrence of fast green corona events during the 20 th cycle is also found to be rather higher than it was in cycle 19 (Demastus et al., 1973).

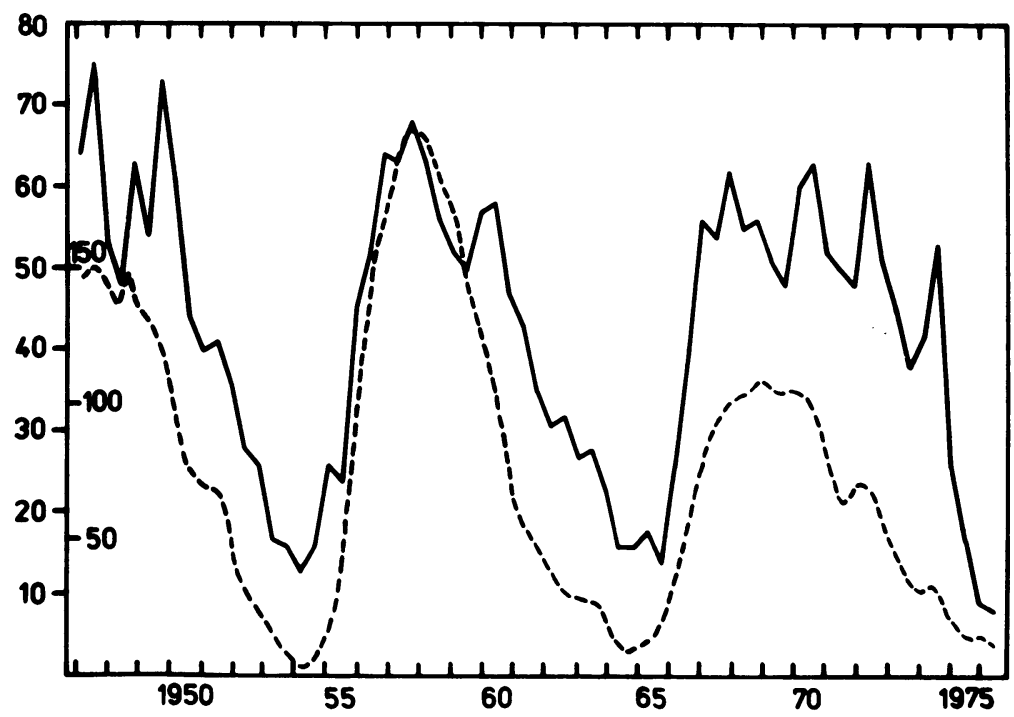

Figure 6. 11-year cycle variations of the green corona (solid line - left hand scale in absolute coronal units) and Wolf numbers (dashed line - right hand scale). 
North-south asymmetry of the solar activity certainly belongs to the long-term properties of the sun, because during long time intervals, months or years, the solar activity remains asymmetrical. It even seems (Waldmeier, 1957b, 1971) that the asymmetry is not connected with the 11-year cycle, but perhaps it is ruled by a cycle with a longer period.

The N-S asymmetry of the green corona, defined as $N-S / N+S$, was mostly positive during the last three cycles, as it was for spots, faculae, prominences, the white light corona, etc. The most outstanding asymmetry (see Figure 7) is seen in the period of 1958-1970 with a maximum in 1966 when, according to Dodson and Hedeman (1969), the N-S asymmetry in sunspots was the highest during the past 100 yrs.

An interesting result was published by Waldmeier (1971) for the period 1959-1969). During this period of outstanding positive asymmetry of the green corona, he found the intensity of the red emission line $(637.4 \mathrm{~nm})$ to be asymmetric in the opposite sense. Applying the theory of dielectric recombination to the found green-red line intensity ratio, from such a behaviour it follows that over the most active hemisphere the corona is really denser and hotter.

In the last decade the question of the existence and reality of two maxima of activity during the 11-year solar cycle was raised.

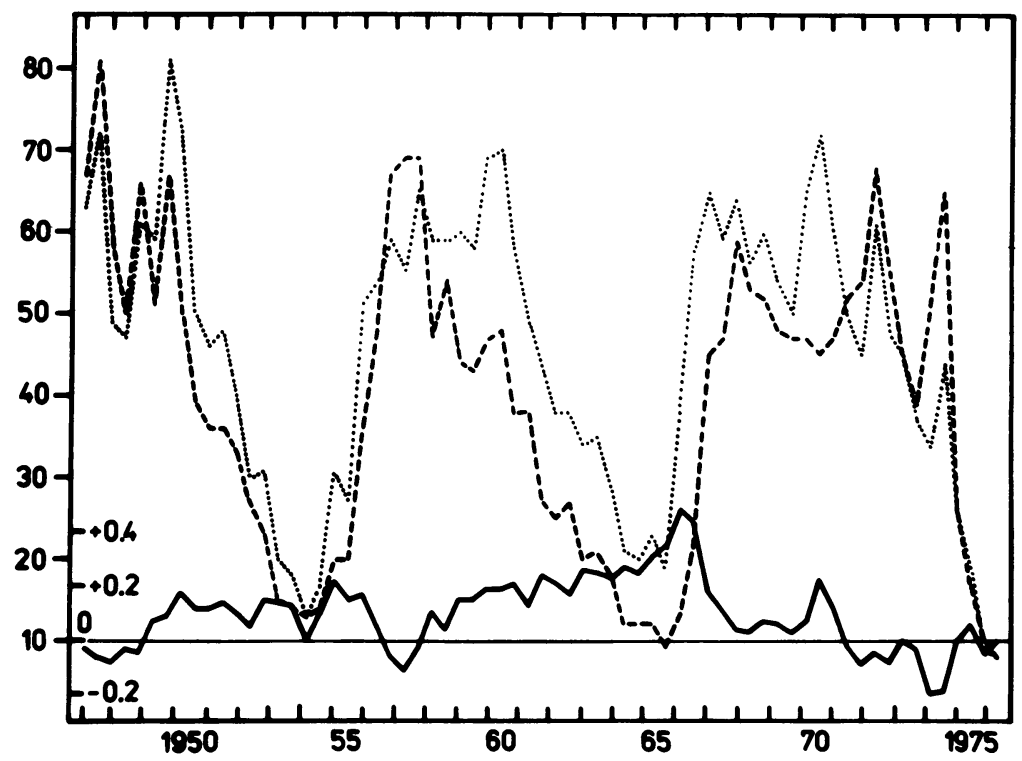

Figure 7. 11-year cycle variation of the green corona intensity, drawn separately for northern (dotted line) and southern (dashed line) hemispheres (left hand scale in absolute coronal units). The $\mathrm{N}-\mathrm{S} / \mathrm{N}+\mathrm{S}$ asymmetry is drawn by the full line (right hand scale)。 


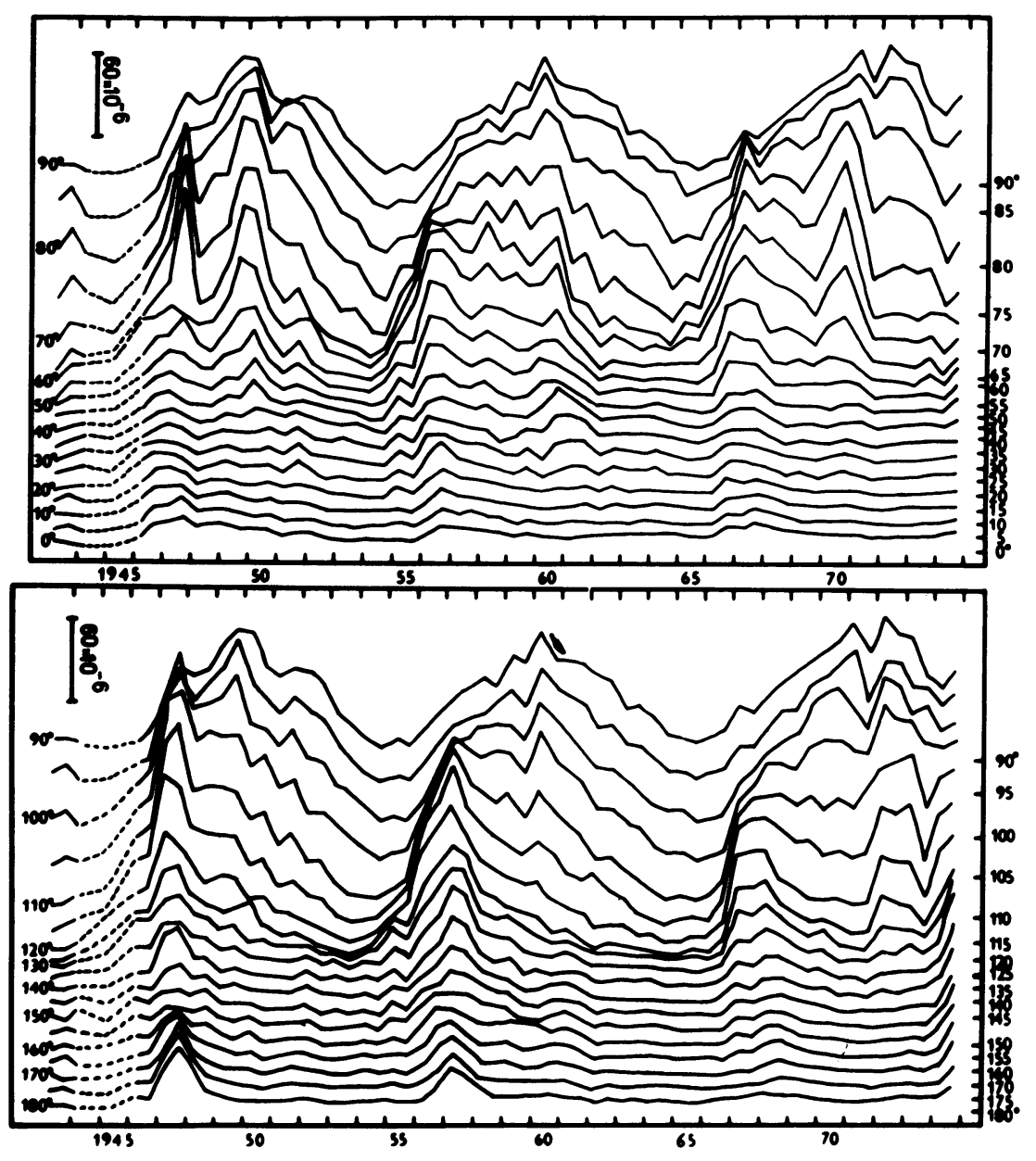

Figure 8. The time dependence of the $530.3 \mathrm{~nm}$ coronal line intensity for different northern (above) and southern (below) latitudes.

In our report this question is pertinent if for no other reason, then because it was first introduced thanks to the study of the time distribution of the coronal emission (Gnevyshev, 1967). Recently it was again summarized (Gnevyshev, 1977) and a list of papers was given in which two maxima have been found for various manifestations of solar and geophysical activity.

Usually two coronal maxima were demonstrated on data of 19th cycle. Some papers declaring the reality of this feature in 20th cycle showed to be premature (Pathak, 1972; Cuperman and Sternlieb, 1972). These authors only had data up to 1971 at their disposal and they evaluated the activities in 1967 and 1969-1970 as the two maxima. But it is generally known and it can clearly be seen in our Figures 
2, 6 and also 8 (constructed from the data of Pic du Midi only, to avoid any possible influence of the non-linear relation of the photo metric scales of different observatories) that there were two subsequent coronal maxima in 1972 and 1974. Because cycle No.18 - the first for which the coronal data are sufficiently complete - does not seem to be very expressive in the sense of the existence of two maxima either, we suggest one should be more careful before declaring this feature to be a "basic feature of the 11-year cycle". From Figure 8, in combination with Figure 3 it follows that: (1) The curves sometimes are not so smooth as they are frequently presented. (2) The activity of the green corona seems to follow the development of the features known as "complexes of activity", the lifetime of which is 1-2 years. (3) This process is relatively independent in both hemispheres.

What we only can mention as some sort of secondary activity at low latitudes just before the solar cycle minima is reality shown in Figure 9, where the largest half-yearly values of the green corona in the whole latitudinal interval $\left( \pm 60^{\circ}\right.$ ) are marked separately for the northern and southern hemispheres. The regular displacement of activity to the equator seems to have been disrupted in 1949, 1961 and 1971. Beyond these years the activity remained at the same or was even shifted to higher latitudes. This may be, but is not necessarily connected with a real increase of the activity level.

\section{CORONAL HOLES}

In dealing with coronal responses to long-time phenomena one should definitely summarize briefly our state-of-knowledge on coro-

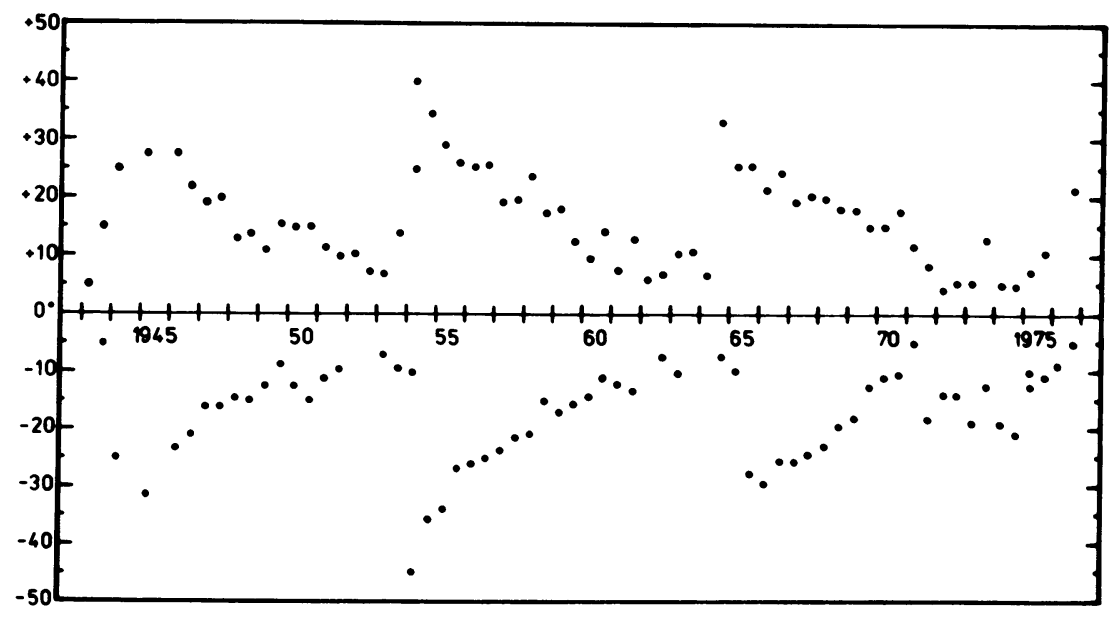

Figure 9. Maximum values of half-yearly averages of the green coronal line intensity. 
nal holes. During the last five years a large number of papers appeared which clearly indicate that the coronal holes belong to the fundamental features of the Sun. From the point of view of our introductory paper their following properties are of principal importance:

(1) Coronal holes are predominantly magnetic features. The photospheric magnetic fields underlying a hole are weak, predominantly unipolar, with an open configuration of the field lines. During the Skylab mission low-latitude holes tended to develop in magnetic cells of the same polarity as the polar cap in that same hemisphere (Bohlin and Sheeley, 1978).

(2) Generally, coronal holes are divided into two groups: polar and low-latitude coronal holes. The first are more extended in size and more stable. For the Skylab period Bohlin (1977) has reported an average of 18 to $19 \%$ of the Sun's total surface covered by coronal holes, of which $15 \%$ was contained in the polar caps. Altschuler et al. (1972) have found $13 \%$ of the Sun's surface covered by lower-latitude holes in November 1966. From a study of coronal holes in the period of 1963-1974 Broussard et al. (1978) were able to draw a picture of the coronal holes variation with the sunspot cycle. The polar coronal holes, prominent at solar minimum, decreased in area as the solar activity increased and were small or absent at maximum phase. During the maximum coronal holes occurred poleward of the sunspot belts and in the equatorial region between them. The observed equatorial holes were small and persisted for one or two solar rotations only. The general conclusion is that the coronal holes wax and wane during the cycle in consistence with most long-term features observed in emission- and K-coronas.

(3) According to Bohlin (1977) the lifetime of holes typically exceeded 5 or more rotations and frequently reached values of $8-10$ rotations. In 1974-1975 one hole or its remnants may have lasted 20 rotations (indications see, for example, in Sheeley et al., 1976).

(4) As we have shown in Section 2 a well-known characteristic of solar corona activity is its tendency to persist in certain preferred zones on the Sun, frequently over many solar rotations. BohIin (1977) claims that the same result seems to hold true for coronal holes. However, on a time-scale of the 8 months of Skylab, there is no longitude zone greater than $40^{\circ}$ in width that did not have a hole at some latitude, north or south. This means that on a long-term average the holes appear uniformly over the solar sphere. The result formulated in this way probably does not take into account the difference in the rotation rates of the corona and photosphere, pointed out in section 4. Sheeley et al. (1976) adopted a better approach to demonstrate the longitudinal distribution of coronal holes. On a sequence of 27-day Bartels rotations for the interval 1973-1975 the arrangement of the holes into two long-term strips is clearly seen, nevertheless, the statement, similar to Bohlin's, that over this 3-year interval some hole occurred at each longitude, is also valid. 
(5) Evolution of the coronal holes through the solar cycle has been discussed in some details by Bohlin and Sheeley (1978) who suppose that the holes form as a result of the emergence of two or more new BMR's which are sufficiently close together to prevent their respective proceding and following magnetic fields (of like sign) from achieving flux balance. This was the case of all four major holes observed during the manned Skylab missions. Iarge-scale shifts in boundary locations were found to explain most of the evolution of coronal holes (Nolte et al., 1978a). The authors conclude that coronal holes evolve by magnetic field lines opening when the holes are groving, and by fields closing as the holes shrink. The disappearence of the large holes near solar minimum and perhaps of the holes in general was hypothesized by filling the area of the holes with many small-scale, magnetically closed features, known as X-ray bright points (Nolte et al., 1978b).

(6) Finally we should mention a fact important, let us say, from the practical point of view. From observations of coronal holes, solar wind streams and geomagnetic disturbances during 1973-1976, Sheeley et al. (1976) conclude, that "the results leave little doubt that coronal holes are related to high-speed streams and their recurrent geomagnetic disturbances". Similarly, "during 1963-1974 whenever XUV or X-ray images were available, nearly all recurrent solar wind streams of speed $\geqslant 500 \mathrm{kms}^{-1}$ were found associated with coronal holes at less than $40^{\circ}$ latitude" (Broussard et al., 1978). On these two quotations it is appropriate to hand word over to the next speaker

\section{REFERENCES}

Altschuler, M.D.: 1974, in G. Newkirk, Jr. (ed.), "Coronal Disturbances", IAU Symp. 57, 3.

Antonucci, E.: 1974, Solar Phys. 34, 471.

Antonucci, E. and Dodero, M.A.: 1977, Solar Phys. 53, 179.

Antonucci, E. and Svalgaard, I.: 1974, Solar Phys. 34, 3.

Antonucci, E. and Svalgaard, L.: 1976, Solar Phys. 36, 155.

Bohlin, J.D.: 1977, Solar Phys. 51, 377.

Bohlin, J.D.and Sheeley, Jr., N.R.: 1978, Solar Phys. 56, 125.

Broussard, R.M., Sheeley, Jr., N.R•, Tousey, R., and Underwood, J.H.: 1978, Solar Phys. 56, 161.

Bumba, V.: 1976, in V. Bumba and J. Kleczek (eds.), "Basic Mechanisms of Solar Activity", IAU Symp. $71,47$.

Bumba, V. and Howard, R.: 1969, Solar Phys. 7, 28.

Bumba, V. and Sýkora, J.: 1973, in M.J. Rycroft and S.K. Runcorn

(eds.), COSPAR Space Research, Vol. XIII, Berlin, p. 1973.

Bumba, V. and Sýkora, J.: 1974, in G. Newkirk, Jr. (ed.), "Coronal

Disturbances", IAU Symp. 57, 73.

Cuperman, So and Sternlieb, A.: 1972, Solar Phys. 25, 493.

Demastus, H.I., Wagner, W.J., and Robinson, R.D.: 1973, Solar Phys. $31,449$.

Dodson, H.W. and Hedeman, E.R.: 1969, IQSY 4, 3. 
Gnevyshev, M.N.: 1967, Solar Phys. 1, 107. Gnevyshev, M.N.: 1977, Solar Phys. 51, 175. Hansen, R.T., Garcia, Ch.J., Hansen, S.F., and Loomis, H.G.: 1969a, Solar Phys. 7, 417.

Hansen, R.T., Hansen, S.F., and Loomis, H.G.: 1969b, Solar Phys. 10, 135.

Howard, R., Bumba, V., and Smith, S.F.: 1967, Carnegie Inst. of Washington Publ. No. 626, Washington.

Knoška, Š., Křivský, I., and Sýkora, J.: 1978, paper presented at IX. Consultation on Solar Physics, Sept. 24 - Oct. 1, 1978, Wroclaw, in press.

Letfus, V., Kulčár, I., and Sýkora, J.: 1980, this Symposium.

Newkirk, Jr., G.: 1971, in R. Howard (ed.), "Solar Magnetic Fields", IAU Symp. 43, 547.

Nolte, J.T., Davis, J.M., Gerassimenko, M., Krieger, A.S., and Solodyna, C.V.: 1978a, Solar Phys. 60, 143.

Nolte, J.T., Gerassimenko, M., Krieger, A.S., and Solodyna, C.V.: 1978b, Solar Phys. 56, 153.

Pathak, P.N.: 1972, Solar Phys. 25, 489.

Pneuman, G.W.: 1974, in G. Newkirk, Jr॰ (ed.), "Coronal Disturbances", IAU Symp. 57, 35 .

Sheeley, Jr., N.R., Harvey, J.W., and Feldman, W.C.: 1976, Solar Phys. 49, 271.

Stenflo, J.0.: 1972, Solar Phys. 23, 307.

Stenflo, J.0.: 1974, Solar Phys. 36, 495.

Stenflo, J.0.: 1977, Astron. Astrophys. 61, 797.

Sýkora, J.: 1971a, Bull. Astron. Inst. Czech. 22, 12.

Sýkora, J.: 1971b, Solar Phys. 18, 72.

Sýkora, J.: 1975, Contr. Astron. Obs. Skalnaté Pleso 5, 5.

Timothy, A.F., Krieger, A.S., and Vaiana, G.S.: 1975, Solar Phys. $42,135$.

Trellis, M.: 1957, Suppl. Ann. Astrophys. No.5.

Wagner, W.F.: 1975, Astrophys. J. 198, L141.

Waldmeier, M.: 1957a, Die Sonnenkorona, Verlag Birkhäuser, Basel.

Waldmeier, M.: 1957b, Z. Astrophys. 43, 149.

Waldmeier, M.: 1971, Solar Phys. 20, 332.

Wilcox, J.M.: 1971, in R. Howard (ed.), "Solar Magnetic Fields",

IAU Symp. 43, 744 .

Wilcox, J.M., Schatten, K.H., Tanenbaum, A.S., and Howard, R.: 1970,

Solar Phys. 14, 255.

Xanthakis, J.: 1969, Solar Phys. 10, 168. 